\title{
DERECHO Y POLÍTICAS AMBIENTALES EN LA REGIÓN DE MURCIA
}

\author{
SANTIAGO M. ÁlVAREZ CARREÑO \\ Profesor titular de Derecho Administrativo \\ Universidad de Murcia \\ ELISA PÉREZ DE los COBOS HERNÁNDEZ \\ Investigadora \\ Departamento de Derecho Administrativo \\ Universidad de Murcia
}


Sumario: 1. Manifestaciones organizativas de la política y legislación ambiental de la Administración regional en Murcia. 1.1. Consejo Asesor Regional de Medio Ambiente. 1.2. Consejerías. 2. Caza y pesca. 2.1. Caza. 2.2. Pesca. 3. Montes. 4. Agricultura y ganadería. 4.1. Agricultura. 4.2. Ganadería. 5. Vías pecuarias. 6. Turismo. 7. Evaluación ambiental de proyectos. 8. Ordenanzas locales.

1. Manifestaciones organizativas de la política y legislación ambiental de la Administración regional en Murcia

\subsection{Consejo Asesor Regional de Medio Ambiente}

En relación con el órgano citado, cabe hacer referencia al Anuncio, de 24 de enero de 2014, de la Consejería de Presidencia por el que se somete a información pública el Proyecto de Decreto por el que se modifican las funciones, composición y régimen de convocatorias del Consejo Asesor Regional de Medio Ambiente.

Los diversos cambios organizativos que en los últimos años han tenido lugar en el Gobierno regional para el ejercicio de las competencias de medio ambiente, hoy atribuidas a la Consejería de Presidencia ${ }^{1}$, han motivado la elaboración de un proyecto de decreto por el que se pretende adecuar la composición del Consejo Asesor Regional de Medio Ambiente, actualizando sus funciones y el régimen de convocatorias, incorporando además la denominación de las consejerías representadas en este órgano que han resultado afectadas por las sucesivas reorganizaciones departamentales y contemplando igualmente que para futuros cambios organizativos no se requiera la modificación de la disposición ${ }^{2}$.

Dada su naturaleza, el referido Proyecto de Decreto se somete a información pública durante un mes en cumplimiento de lo establecido en el artículo 53. 4 de la Ley 6/2004, de 28 de diciembre, del Estatuto del Presidente y del Consejo de Gobierno, así como en el artículo 16 de la Ley 27/2006, de 18 de julio, por la que se regulan los derechos de acceso a la información, de participación pública y de acceso a la justicia en materia de medio ambiente, a fin de que cualquier persona física o jurídica pueda presentar por escrito alegaciones y aportar documentos u otros elementos de juicio.

\footnotetext{
${ }^{1}$ De conformidad con lo dispuesto en el Decreto del Presidente 24/2011, de 28 de junio, por el que se establece el orden de prelación de las Consejerías de la Administración Regional y sus competencias.

${ }^{2}$ El Proyecto de Decreto por el que se modifican las funciones, composición y régimen de convocatorias del Consejo Asesor Regional de Medio Ambiente se encuentra disponible en $<$ http://www.murcianatural.carm.es/> (fecha de última consulta: 30/05/2014).
} 
Entre las modificaciones propuestas destacan las referidas al artículo 2 del Decreto 42/1994, de 8 de abril, por el que se regula el Consejo Asesor Regional de Medio Ambiente, en el que se recogen las funciones que corresponden a este órgano, atribuyéndole, entre otras, la de emitir informe sobre los anteproyectos de ley y proyectos de reglamento con incidencia medioambiental; asesorar sobre los planes y programas de ámbito regional que la presidencia del Consejo le proponga en razón a la importancia de su incidencia sobre el medio ambiente; informar en los términos previstos en la legislación vigente los planes de ordenación de los recursos naturales, los planes de gestión de los espacios de la red Natura 2000 o, en su caso, los instrumentos de planificación integrados, así como la declaración de áreas y espacios protegidos; informar el anteproyecto de presupuestos de la Comunidad Autónoma de la Región de Murcia en materia de medio ambiente; emitir informes y efectuar propuestas en materia medioambiental, a iniciativa propia o a petición de las consejerías que así lo soliciten a la presidencia del Consejo; proponer medidas que incentiven la creación de empleo ligado a actividades relacionadas con la protección del medio ambiente, así como la participación ciudadana en la solución de los problemas ambientales; proponer medidas de educación ambiental que tengan como objetivo informar, orientar y sensibilizar a la sociedad de los valores ecológicos y medioambientales; e impulsar la coordinación entre la iniciativa pública y privada en materia de medio ambiente.

Asimismo, y por lo que se refiere a su composición, se hace pública la intención de modificar el artículo 3 del Decreto 42/1994, concretando que el Consejo Asesor Regional de Medio Ambiente quedará integrado por el presidente, el consejero de Presidencia o el titular del departamento con competencias en medio ambiente, el vicepresidente, el director general de Medio Ambiente o el titular del centro directivo con competencias en medio ambiente, y los vocales ${ }^{3}$.

\footnotetext{
${ }^{3}$ Serán vocales del Consejo Asesor Regional de Medio Ambiente: i) un representante de cada una de las siguientes consejerías designado por los titulares de estas: a) Obras Públicas y Ordenación del Territorio, o departamento con competencias en ordenación del territorio; b) Universidades, Empresa e Investigación, o departamento con competencias en materia de industria, energía y minas; c) Sanidad y Política Social, o departamento con competencias en sanidad; d) Agricultura y Agua, o departamento con competencias en materia de agricultura; ii) un representante de cada una de las universidades de la Región de Murcia; iii) un representante del Centro Oceanográfico de Murcia del Instituto Español de Oceanografía; iv) un representante del Centro de Edafología y Biología Aplicada del Segura del Consejo Superior de Investigaciones Científicas; v) tres representantes de las asociaciones ecologistas y protectoras de la naturaleza registradas como tales en el Registro de Asociaciones Ecologistas y Protectoras de la Naturaleza de la Región de Murcia designados por y entre ellas; vi) dos representantes de las organizaciones empresariales más representativas en la Región de Murcia elegidos por estas; vii) un representante de la organización profesional agraria más representativa en la Región de Murcia; viii)
} 
Por último, queda prevista la modificación del artículo 6, referido a las convocatorias, estableciéndose que el Pleno se reunirá con carácter ordinario una vez al cuatrimestre como mínimo y siempre que deba informar sobre asuntos de su competencia. Asimismo, se reunirá cuando lo soliciten al menos un tercio de sus vocales para tratar los temas planteados por ellos, debiendo en este caso ordenarse la convocatoria por el presidente para su celebración en el plazo máximo de quince días.

\subsection{Consejerías}

En primer lugar, debemos referirnos a la reorganización de la Administración regional mediante el Decreto de la Presidencia 4/2014, de 10 de abril, de reorganización de la Administración Regional.

El presente Decreto introduce modificaciones sustanciales en cuanto al número, la denominación y las competencias de las distintas consejerías de la Región de Murcia, efectuando una nueva distribución competencial entre los departamentos de la Administración regional que obliga, como se examina a continuación, a establecer los órganos directivos de las nuevas consejerías mediante los correspondientes decretos del Consejo de Gobierno.

En este sentido, el artículo 1 del Decreto de referencia establece que la Administración de la Comunidad Autónoma de la Región de Murcia se organiza en las consejerías que a continuación se citan y con el orden de prelación que asimismo se indica: Consejería de Presidencia y Empleo; Consejería de Economía y Hacienda; Consejería de Agricultura y Agua; Consejería de Industria, Turismo, Empresa e Innovación; Consejería de Educación, Cultura y Universidades; Consejería de Fomento, Obras Públicas y Ordenación del Territorio; y Consejería de Sanidad y Política Social.

Visto lo anterior, quedan derogados los decretos del Presidente núm. 13/2013, de 23 de julio, de reorganización de la Administración Regional, y núm. 17/2013, de 25 de julio,

un representante de las cámaras de Comercio, Industria y Navegación de la Región de Murcia elegido por estas; ix) un representante elegido por cada uno de los sindicatos más representativos en la Región de Murcia; $\mathrm{x}$ ) un representante de los ayuntamientos de la Región de Murcia designado por la Federación de Municipios de la Región de Murcia; xi) un representante de la organización de consumidores y usuarios más representativa de la Región de Murcia; xii) un representante de las comunidades de regantes de la Región de Murcia elegido por estas; xiii) un representante de la Federación de Asociaciones de Vecinos de la Región de Murcia; xiv) dos representantes de las asociaciones de propietarios forestales y/o de las asociaciones de propietarios de terrenos en espacios naturales protegidos de la Región de Murcia elegidos por y entre ellas; xv) y un representante de la Federación de Caza de la Región de Murcia. 
por el que se establece el orden de prelación de las Consejerías de la Administración Regional y sus competencias.

Por otro lado, hay que hacer mención al Decreto 42/2014 de Consejo de Gobierno, de 14 de abril, por el que se establecen los órganos directivos de la Consejería de Agricultura y Agua. La Consejería de Agricultura y Agua es el departamento de la Comunidad Autónoma de la Región de Murcia encargado de la propuesta, el desarrollo y la ejecución de las directrices generales del Consejo de Gobierno en materia de agricultura, desarrollo rural, ganadería, industria agroalimentaria, pesca y agua, así como las que la normativa vigente le atribuye como organismo autorizado para el pago de los gastos correspondientes a la Política Agraria Común (organismo pagador); las relativas a la investigación y el desarrollo tecnológico en los sectores agrario, forestal y alimentario; la investigación en materia de pesca, marisqueo, acuicultura marina, alguicultura y cualquier otra forma de cultivo industrial, medio ambiente, así como cualesquiera otras que le asigne la legislación vigente.

A la luz de las modificaciones introducidas mediante el Decreto de la Presidencia 4/2014, de 10 de abril, de reorganización de la Administración Regional, el presente Decreto 42/2014, de 14 de abril, viene a establecer los órganos directivos de esta consejería con el fin de facilitar el desarrollo de los cometidos y las competencias que le han sido atribuidos: Secretaría General y Vicesecretaría; Dirección General de Industria Agroalimentaria y Capacitación Agraria; Dirección General de Regadíos y Desarrollo Rural; Dirección General de Ganadería y Pesca; Dirección General del Agua; Dirección General para la Política Agraria Común; Dirección General de Medio Ambiente; Subdirección General de Calidad Ambiental; y Subdirección General de Medio Natural.

Quedan adscritos a esta consejería el organismo autónomo Instituto Murciano de Investigación y Desarrollo Agrario y Alimentario (IMIDA) y la Entidad de Saneamiento y Depuración de Aguas Residuales de la Región de Murcia (ESAMUR).

\section{Caza y pesca}

\subsection{Caza}

En primer lugar, debe mencionarse la Orden de 9 de mayo de 2014, de la Consejería de Agricultura y Agua, sobre períodos hábiles de caza para la temporada 2014/2015 en la Comunidad Autónoma de la Región de Murcia. 
Dentro del régimen jurídico básico establecido en la Ley 42/2007, de 13 de diciembre, del Patrimonio Natural y de la Biodiversidad, relativo a la actividad cinegética, la presente Orden, dictada en desarrollo del artículo 42.1 de la Ley 7/2003, de 12 de noviembre, de Caza y Pesca Fluvial de la Región de Murcia, establece para la temporada cinegética 2014/2015 los períodos hábiles (art. 3), las modalidades de caza (arts. 5, 6 y 7) y las zonas y las especies susceptibles de aprovechamiento cinegético (art. 2).

Desde el punto de vista ambiental, se pretende garantizar la conservación del patrimonio natural existente en la Comunidad Autónoma de Murcia compatibilizando la gestión de los recursos cinegéticos con la protección del hábitat de nuestra fauna silvestre, destacando las medidas de protección de determinadas especies y de la caza en general (art. 8) y la regulación que se contiene de la actividad cinegética en espacios naturales (disposición adicional 1. ${ }^{\mathrm{a}}$ ).

Por otro lado, hay que hacer referencia a la presentación, en fecha 30 de mayo de 2014, del protocolo de actuaciones para alcanzar un nuevo modelo de gestión del arruí en la Región de Murcia.

La introducción del arruí (Ammotragus lervia) en Murcia tuvo lugar en la Reserva Nacional de Caza de Sierra Espuña en 1973 por iniciativa del ICONA. Con el paso del tiempo, la especie amplió su población y su área de distribución a zonas más o menos alejadas de su núcleo inicial. Actualmente ocupa entre 60.000 y 70.000 hectáreas, distribuidas por el centro y oeste de la Región, y se ha venido cazando en los últimos trece años en unos 130 cotos. El crecimiento de la población de arruí ha consolidado la demanda cinegética desde el colectivo de cazadores, pero también ha ocasionado problemas en el entorno, con afectaciones sobre la flora protegida y daños continuados sobre cultivos agrícolas de la periferia de sierra Espuña y del resto del área de distribución. Desde hace unos meses, dada la situación de sequía acumulada, se viene detectando un incremento en las demandas de agricultores y propietarios de dicho entorno por los daños ocasionados.

Por todo ello, la Dirección General de Medio Ambiente presentó el pasado 30 de mayo de 2014 ante los agentes sociales un protocolo de actuaciones para alcanzar un nuevo modelo de gestión del arruí en la Región que mejore la situación actual y solucione la 
problemática planteada por el crecimiento excesivo de la población de esta especie exótica ${ }^{4}$.

Medio Ambiente admite por primera vez que la Reserva Regional de Caza es deficitaria para la Administración y que apenas aporta beneficios al entorno. También por primera vez se advierte de forma oficial sobre los daños en la vegetación protegida y el freno que supone para la expansión de la cabra montés, el ungulado autóctono. Este cambio de paradigma coincide con la reubicación de las competencias de medio ambiente en la Consejería de Agricultura y Agua, desde la que se pone de relieve también la especial circunstancia de que Reserva de Caza y Parque Regional "se superponen en el territorio" y que es necesario dar preferencia a la "conservación de los ecosistemas y los recursos faunísticos". Los cambios en la gestión del arruí también afectan a la dirección técnica de la Reserva de Caza, que pasa a depender del director-conservador del Parque, cuando tradicionalmente se había administrado desde el Servicio de Biodiversidad, Caza y Pesca Fluvial ${ }^{5}$.

Según la información facilitada desde la CARM, el planteamiento general de Medio Ambiente es reducir el número de ejemplares en sierra Espuña a 300 (como se especifica en el Plan de Ordenación de los Recursos Naturales del Parque Regional, una intención anunciada ya hace casi dos años pero no llevada a la práctica) ${ }^{6}$; fijar las poblaciones restantes en el interior de la Reserva; controlar drásticamente a la especie en el resto de la Región (se distribuye por el centro, el oeste y algunos puntos del noroeste de la Comunidad Autónoma, y se caza en 130 cotos desde hace 13 años); y definir en qué zonas de la Región debe quedar localizado el arruí y con cuántos ejemplares, con un modelo de funcionamiento que responda a "las nuevas normativas surgidas en materia de gestión de espacios naturales, especies exóticas, conservación de hábitats y planificación cinegética". A fin de lograr estos objetivos, se ha decidido un plan de actuación en tres niveles en el que se distinguen actuaciones urgentes y a corto

\footnotetext{
${ }^{4}$ Información disponible en $<$ http://www.carm.es/web/pagina?IDCONTENIDO=75247\&IDTIPO=10\&RASTRO=c $\$$ m $122,70>($ fecha de última consulta: 01/06/2014).

${ }^{5}$ Cfr. <http://lospiesenlatierra.laverdad.es/noticias/3069-medio-ambiente-se-plantea-eliminar-el-arrui-ensierra-espuna>.

${ }^{6} \mathrm{Cfr} .<\mathrm{http}$ //lospiesenlatierra.laverdad.es/noticias/2471-solo-pueden-quedar-300>.
} 
plazo (4 meses), medidas a medio plazo (5-12 meses) y medidas a largo plazo (12-24 meses) $)^{7}$.

\subsection{Pesca}

Aquí debemos tener en cuenta la Orden de 11 de noviembre de 2013, de la Consejería de Agricultura y Agua, por la que se establece un período de veda temporal para el ejercicio de la pesca de cerco en aguas interiores de la Región de Murcia.

Esta orden declara una veda temporal para el ejercicio de la pesca en la modalidad de cerco en aguas interiores de la Región de Murcia durante el período comprendido entre los días 6 de diciembre de 2013 y 6 de enero de 2014, ambos inclusive.

De este modo, la Consejería de Agricultura y Agua, en el marco facilitado por el artículo 32 del Real Decreto 1549/2009, de 9 de octubre, sobre ordenación del sector pesquero y adaptación al Fondo Europeo de la Pesca $^{8}$, continúa con la línea de medidas adoptadas para limitar el esfuerzo pesquero de su flota a través del establecimiento de períodos temporales de veda con el fin de garantizar la plena efectividad del Plan Integral de Gestión del Mediterráneo 9 .

\footnotetext{
${ }^{7}$ Entre las medidas a corto plazo destacan la reducción de la población en el Parque Regional de Sierra Espuña a 300 ejemplares; el mantenimiento de vallado para evitar la dispersión de los animales y para que se aquerencien a zonas no conflictivas. Impulso de medidas para evitar o compensar daños; aumentar el control de la caza en cotos situados en el interior del Parque y zonas colindantes con el espacio protegido; trasladar competencias de la gestión de la Reserva de Caza al director-conservador del Parque Regional; y dinamizar el funcionamiento del Grupo de Trabajo del Arruí. Se propone la medida excepcional de abatimiento para el control de la población y erradicación en terrenos libres. Por su parte, las medidas a medio plazo contemplan la reducción de las poblaciones de arruí en el resto del área de distribución de la especie, así como la elaboración de un plan regional de gestión que defina una zona de confinamiento de la especie. Por último, las medidas a largo plazo plantean definir un nuevo modelo de gestión del arruí en el Parque Regional de Sierra Espuña y la regulación intensa de la caza de esta especie en la Región a través de los planes de ordenación cinegética en los cotos del área de confinamiento que se establezca y su eliminación en el resto de la Región. Información disponible en: $<\mathrm{http}: / /$ www.carm.es/web/pagina?IDCONTENIDO=75247\&IDTIPO=10\&RASTRO=c\$m122,70> (fecha de última consulta: 1/06/2014).

${ }^{8}$ El artículo 32 del Real Decreto 1549/2009, de 9 de octubre, sobre ordenación del sector pesquero y adaptación al Fondo Europeo de la Pesca (BOE, núm. 245, de 10 de octubre de 2009), contempla la posibilidad de efectuar paradas temporales en las épocas más adecuadas desde el punto de vista biológico para la recuperación de las especies afectadas por un plan de los previstos en el artículo 21 del Reglamento (CE) núm. 1198/2006 del Consejo, de 27 de julio.

${ }^{9}$ Orden AAA/2808/2012, de 21 de diciembre, por la que se establece un Plan de Gestión Integral para la conservación de los recursos pesqueros en el Mediterráneo afectados por las pesquerías realizadas con redes de cerco, redes de arrastre y artes fijos y menores, para el período 2013-2017 (BOE, núm. 313, de 29 de diciembre de 2012).
} 
Por otro lado, hay que dejar constancia de la Orden de 30 de abril de 2014, de la Consejería de Agricultura y Agua, por la que se regula la pesca artesanal del pulpo (Octopus vulgaris) en aguas de la Comunidad Autónoma de la Región de Murcia.

Hacíamos mención en la crónica anterior a la Orden de 27 de junio de 2013, de la Consejería de Agricultura y Agua, por la que se modificaba la Orden de 19 de septiembre de 2002, por la que se regula la pesca artesanal de pulpo (Octopus vulgaris) con nasas en aguas interiores de la Región de Murcia, señalando la necesaria ordenación de esta pesca artesanal de pulpo como un factor imprescindible para la conservación de la especie objetivo y para evitar posibles interacciones con otras pesquerías ${ }^{10}$. Lo anterior, junto con la necesidad de proporcionar una normativa que contemple de forma integral todos los aspectos relativos a este tipo de pesquería, conducen al dictamen de esta nueva Orden que, derogando la anterior de 2002, viene a incluir en su articulado el arte de pesca denominado cadufo o alcatruz ${ }^{11}$, no contemplado hasta este momento.

La Orden tiene por objeto regular la pesca de pulpo practicada por la flota de artes menores en aguas del litoral de la Región de Murcia —ampliando su ámbito de aplicación a las aguas exteriores ${ }^{12}$ - con los artes de trampa regulados en esta, excluyendo la captura del pulpo con artes de red y anzuelo específicamente reglamentados practicada por embarcaciones profesionales de pesca.

Entre otras novedades, como la ampliación del período de veda (art. 7) o la identificación y señalización de los artes (art. 10), la Orden incluye, ex artículo 13, la utilización de otros artes de trampa como el cadufo, cuyo uso regula en los aspectos esenciales.

También hay que hacer mención de la Orden de 8 de mayo de 2014, de la Consejería de Agricultura y Agua, por la que se regula el calamento del arte de langostinera en el Mar Menor. El arte de langostinera es utilizado en el Mar Menor para la captura del langostino en determinadas épocas del año, una especie que alcanza un gran valor

\footnotetext{
${ }^{10}$ Cfr. ÁlVAReZ CARReÑO, S. M. y SALAZAR ORTUÑO, E., "Derecho y Políticas Ambientales en la Región de Murcia”, RCDA, vol. 4, núm. 2, 2013 (accesible en $<$ http://www.rcda.cat/index.php/rcda/article/viewFile/416/1973>. Último acceso: 29/05/2014).

${ }^{11}$ Los cadufos o alcatruces son útiles de pesca constituidos por un recipiente de arcilla que permite la entrada y salida de la especie objetivo. Cfr. artículo 13 Orden de 30 de abril de 2014, de la Consejería de Agricultura y Agua, por la que se regula la pesca artesanal del pulpo (Octopus vulgaris) en aguas de la Comunidad Autónoma de la Región de Murcia.

${ }^{12}$ Artículo 21 de la Ley 2/2007, de 12 de marzo, de Pesca Marítima y Acuicultura de la Región de Murcia.
} 
económico. El sector pesquero ha venido demandando la modificación de aspectos puntuales de esta pesquería, lo que ha conllevado una regulación más detallada de esta dentro del marco del Reglamento de Pesca en el Mar Menor.

La Orden tiene por objeto la regulación del calamento del arte de langostinera también llamado charamita - utilizado para la pesca del langostino y contemplado en el Decreto 91/1984, de 2 de agosto, por el que se aprueba el Reglamento de Pesca en el Mar Menor.

Entre los aspectos regulados por la Orden destacan los períodos de calamento (art. 2), las zonas y artes autorizados (art. 3), las autorizaciones necesarias (art. 4) o la identificación y señalización de las artes (art. 5).

Finalmente, debemos mencionar la Orden de 22 de mayo de 2014, de la Consejería de Agricultura y Agua, por la que se prorroga la veda para la pesca de moluscos bivalvos en aguas interiores de la Región de Murcia establecida por la Orden de 16 de junio de 2005 .

Los bivalvos son una clase del filo Mollusca con unas 13.000 especies, generalmente marinos. Presentan un caparazón con dos valvas laterales, generalmente simétricas, unidas por una bisagra y ligamentos. Constituyen ejemplos representativos de este grupo la chirla, la vieira, la ostra, los mejillones, los dátiles de mar, etc. ${ }^{13}$

La Orden prorroga por un plazo de tres años, hasta el 28 de junio de 2017, la vigencia de la veda establecida para la pesca de moluscos bivalvos en aguas interiores de la Región de Murcia dispuesta por la Orden de 16 de junio de 2005, de la Consejería de Agricultura y Agua $^{14}$, al no resultar modificadas las circunstancias que motivaron el establecimiento de la veda.

\footnotetext{
${ }^{13}$ Información disponible en $<$ http://www.regmurcia.com/servlet/s.Sl?sit=c,365,m,108\&r=ReP-27467DETALLE_REPORTAJESABUELO $>$ (Fecha de última consulta: 01/06/2014).

${ }^{14}$ Por Orden de 16 de junio de 2005, de la Consejería de Agricultura y Agua (BORM, núm. 145, de 27 de junio de 2005), se aprobó una veda temporal de tres años para la pesca de moluscos bivalvos en aguas interiores en las zonas de producción declaradas por la Orden de 29 de mayo de 1998. Dicha veda ha sido prorrogada por plazos de igual duración mediante la Orden de 26 de junio de 2008, de la Consejería de Agricultura y Agua (BORM, núm. 155, de 5 de julio de 2008), y la Orden de 30 de mayo de 2011 (BORM, núm. 126, de 3 de junio de 2011) al no haberse manifestado en estas signos de recuperación tras los estudios de seguimiento realizados.
} 


\section{Montes}

En relación con las quemas agrícolas y forestales, debe tenerse en cuenta la Resolución de la Dirección General de Medio Ambiente por la que se amplía para el año 2014 el período de peligro y se suspenden la vigencia y los efectos de las autorizaciones para quemas emitidas de conformidad con la Orden de 24 de mayo de 2010, de la Consejería de Agricultura y Agua, sobre medidas de prevención de incendios forestales en la Región de Murcia para el año 2010.

El pasado 23 de abril de 2014 el Servicio de Planificación, Áreas Protegidas y Defensa del Medio Natural de la Dirección General de Medio Ambiente emitió un informe por el que, alertando de las especiales y desfavorables condiciones meteorológicas de los últimos meses en la Región de Murcia, así como las experimentadas en la estación de primavera, declaraba necesario acordar la suspensión temporal de la vigencia y los efectos de las autorizaciones ya emitidas para quemas sobre la base de la Orden de 24 de mayo de 2010, de la Consejería de Agricultura y Agua, sobre medidas de prevención de incendios forestales para el año $2010^{15}$, hasta que las condiciones adversas desaparezcan.

Del mismo modo, dadas las condiciones adversas de sequía y estrés hídrico del combustible forestal, lo que favorece de manera importante la evolución negativa de un posible conato de incendio, la Resolución modifica el período de peligro (artículo 3 de la mencionada Orden), habiéndose propuesto por el Servicio de Planificación, Áreas Protegidas y Defensa del Medio Natural de la Dirección General de Medio Ambiente el adelanto de este, incluyendo para el año 2014 el mes de mayo, por lo que la época de peligro queda establecida desde el 1 de mayo hasta el 30 de septiembre.

\footnotetext{
${ }^{15}$ Con fecha 28 de mayo de 2010 se publica en el BORM la Orden de 24 de mayo de 2010, de la Consejería de Agricultura y Agua, sobre medidas de prevención de incendios forestales para el año 2010 (BORM, núm. 121, de 28 de mayo), cuya vigencia resulta automáticamente prorrogada en los sucesivos años hasta el presente, de conformidad con lo dispuesto en su disposición adicional cuarta. El artículo 9 de la citada Orden prohíbe con carácter general la realización de quemas agrícolas y forestales en la época de peligro, excepcionándose dentro de este período, previa autorización, y salvo los meses de julio y agosto, las quemas que tengan por objeto prevenir o paliar daños causados por plagas o enfermedades, o evitar otros riesgos de mayor gravedad, bajo medidas extraordinarias de prevención.
} 


\section{Agricultura y ganadería}

\subsection{Agricultura}

Debe anotarse, en primer lugar, la Orden de 19 de noviembre de 2013, de la Consejería de Agricultura y Agua, por la que se aprueba el pliego de condiciones de la indicación geográfica protegida "Murcia" inscrito en el Registro Comunitario de Denominaciones de Origen Protegidas e Indicaciones Geográficas Protegidas.

Presentada por la Asociación Vino de la Tierra de Murcia la documentación relativa a la solicitud de modificación del Pliego de Condiciones de la IGP "Murcia", inscrito en el Registro Comunitario de Denominaciones de Origen Protegidas e Indicaciones Geográficas Protegidas, la Orden de 19 de noviembre de 2013, de la Consejería de Agricultura y Agua, aprueba la modificación del Pliego de Condiciones de la IGP "Murcia", una vez superado el período de oposición nacional, habiendo emitido Orden de decisión favorable a dicha modificación ${ }^{16}$ y siendo coherente el documento único, que no se modifica, con el pliego de condiciones ${ }^{17}$.

La Consejería de Agricultura y Agua reconoce de este modo la indicación geográfica "Murcia" para los vinos con derecho a la mención tradicional "Vino de la Tierra", lo que permitirá que los vinos de mesa que cuenten con la citada mención dispongan de un sistema de protección de calidad que hasta ahora era exclusivo de los vinos con denominación de origen.

Los vinos designados con esta indicación geográfica protegida proceden exclusivamente de viñedos inscritos en el Registro Vitícola de la Comunidad Autónoma de la Región de Murcia e incluidos en los términos municipales, pagos y pedanías siguientes:

- Términos municipales: Abanilla, Bullas, Cartagena, Cehegín, Fortuna, Fuente Álamo, Jumilla, La Unión, Mula, Pliego, Ricote, Torre Pacheco y Yecla.

- Pagos y pedanías: del término municipal de Calasparra, los pagos comprendidos entre el río Segura y el límite administrativo de los términos de Cehegín y Mula;

\footnotetext{
${ }^{16}$ Orden de 14 de enero de 2013, de la Consejería de Agricultura y Agua, por la que se emite decisión favorable a la solicitud de modificación del pliego de condiciones de la Indicación Geográfica Protegida "Murcia" inscrita en el registro comunitario de Denominaciones de Origen Protegidas y de Indicaciones Geográficas Protegidas (BORM, núm. 22, de 28 de enero de 2013).

${ }^{17}$ El pliego de condiciones y el documento único pueden ser consultados en la dirección electrónica: $<$ https://www.carm.es/web/pagina?IDCONTENIDO=24215\&IDTIPO=11\&RASTRO=c214\$m1 185,3470 2.> (último acceso: 30/05/2014).
} 
del término municipal de Caravaca, los pagos Arrabal de la Encarnación, Campo Coy, Cañada de la Sima y Cañada Lengua; del término municipal de Moratalla, las pedanías de Benizar, Otos y Mazuza, el pago de Las Cañadas y los pagos comprendidos entre la Rambla de Ulea y el límite administrativo de los términos municipales de Cehegín y Calasparra; del término municipal de Lorca, las pedanías de Avilés, Coy, Doña Inés, La Paca y Zarzadilla de Totana; de los términos de Cieza y Totana, los viñedos comprendidos entre estos términos y los de Ricote y Mula y con Lorca y Mula respectivamente.

Además, se incluyen el resto de los viñedos de la Región de Murcia inscritos a fecha 3 de noviembre de 2009 en el Registro Vitícola y los que puedan inscribirse en el futuro siempre que sean de secano y ubicados en otras zonas distintas de las anteriores

Según la normativa establecida, únicamente los vinos elaborados, almacenados y embotellados en las bodegas que dispongan de la certificación oportuna podrán presentarse en el mercado bajo la indicación geográfica "Murcia", que incluye varias tipologías: blanco rosado y tinto, de aguja blanco y rosado, de aguja tinto, de licor y, por último, de uva sobremadurada blanco y rosado y de uva sobremadurada tinto.

\subsection{Ganadería}

En esta materia, es de destacar el Decreto 1/2014, de 17 de enero, del Consejo de Gobierno de la Comunidad Autónoma de la Región de Murcia, por el que se establece la ordenación sanitaria y zootécnica de las explotaciones avícolas de la Región de Murcia.

En el panorama actual de la Región de Murcia, tanto la producción de carne de ave como el sector de la avicultura de puesta destacan como actividades ganaderas de notable relevancia, dinamismo y trascendencia profesional, habiendo proliferado especialmente las explotaciones avícolas centradas en la cría de pollo para carne, conocido como broiler, seguidas a gran distancia por el resto de las producciones avícolas.

Desde el punto de vista sanitario, la incidencia de enfermedades en las explotaciones avícolas y los efectos económicos derivados de estas aconsejan evitar altas concentraciones de aves de corral en la misma zona mediante la limitación de su capacidad. La intensificación de las explotaciones avícolas y su concentración en 
determinados municipios de la región plantean la necesidad de compatibilizar la creación de riqueza originada por esta actividad pecuaria con la exigencia de protección del medio ambiente y de prevención de los efectos sanitarios adversos que pudiera generar la ganadería intensiva.

De este modo, vista la importancia de este sector en la Región de Murcia, el Decreto 1/2014, de 17 de enero, pretende dar respuesta a dos cuestiones fundamentales. En primer término, a la necesidad de actualizar la variada legislación en vigor adecuándola a la realidad productiva, social y económica del sector avícola existente en la Región, desarrollando un nuevo marco normativo sobre la ordenación sanitaria y zootécnica de las explotaciones avícolas y salas de incubación en el ámbito territorial de la Región de Murcia. En segundo lugar, pretende instrumentar en la Comunidad Autónoma la aplicación de la normativa estatal relativa a la ordenación avícola ${ }^{18}, y$, en ejercicio de la competencia exclusiva que esta tiene atribuida en materia de ganadería ex artículo 10.1.6 de su Estatuto de Autonomía, regular el Registro Regional de Explotaciones Avícolas, el procedimiento para la autorización e inscripción de las explotaciones avícolas de nueva instalación y de la ampliación, los cambios de orientación productiva y los cambios de titularidad de las existentes, el Libro de Registro de Explotación y la identificación de las aves de corral.

De este modo, a través de este extenso decreto - distribuido en 18 artículos, una disposición adicional, cuatro disposiciones transitorias, una disposición derogatoria, dos disposiciones finales y un total de once anexos, que ocupan un total de 50 páginas del BORM - se establece una norma de ordenación de las explotaciones avícolas y salas de incubación ubicadas en la Región de Murcia, cuyo objeto queda centrado en la

\footnotetext{
${ }^{18}$ Entre la que debe ser tenida especialmente en cuenta la Ley 8/2003, de 24 de abril, de Sanidad Animal, cuyo artículo 36.1 establece la obligatoriedad de disponer de la autorización previa de la autoridad competente para las explotaciones de nueva instalación o la ampliación de las ya existentes, lo que conllevó la elaboración de una normativa a nivel nacional que se plasmó en el Real Decreto 1084/2005, de 16 de septiembre, de Ordenación de la Avicultura de Carne, modificado por Real Decreto 692/2010, de 20 de mayo, por el que se establecen las normas mínimas para la protección de los pollos destinados a la producción de carne y se modifica el Real Decreto 1047/1994, de 20 de mayo, relativo a las normas mínimas para la protección de terneros. Asimismo, la citada Ley establece en su artículo 38.1 la obligación de todas las explotaciones de animales de estar registradas en la Comunidad Autónoma en que radiquen, por lo que es necesario crear en la Región de Murcia el Registro Regional de Explotaciones Avícolas, integrado en el Registro General de Explotaciones Ganaderas (REGA), regulado mediante Real Decreto 479/2004, de 26 de marzo, por el que se establece y regula el Registro General de Explotaciones Ganaderas. Además de lo anterior, esta nueva regulación debe adaptarse a los requisitos establecidos en el Real Decreto 372/2003, de 28 de marzo, por el que se establece y regula el Registro General de Establecimientos de Gallinas Ponedoras.
} 
regulación del Registro Regional de Explotaciones Avícolas (art. 5), los procedimientos de autorización e inscripción de nuevas explotaciones (arts. 6 y 12), de ampliaciones y cambios de orientación productiva y de inscripción de los cambios de titularidad (arts. 7, 8 y 9), el Libro de Registro de Explotación (arts. 14 y 16) y la identificación de las aves de corral (art. 13). Igualmente, se establecen las condiciones mínimas de ubicación de las explotaciones y su infraestructura zootécnico-sanitaria y de bioseguridad (arts. 3 y 4), derogando para ello el anterior Decreto 14/1995, de 31 de marzo, por el que se dictan normas para la ordenación sanitaria y zootécnica de las explotaciones avícolas y salas de incubación en la Región de Murcia, y modificando el apartado sexto y el anexo III de la Orden de 26 de diciembre de 1997, de la Consejería de Medio Ambiente, Agricultura y Agua, por la que se regula la identificación de las diversas especies ganaderas en la Región de Murcia.

\section{Vías pecuarias}

Aquí debe dejarse constancia de la Resolución de 1 de abril de 2014, de la Consejería de Presidencia, por la que se dicta la instrucción para la realización de determinados procedimientos relativos a la conservación y defensa de vías pecuarias.

Las vías pecuarias constituyen un legado histórico que en el pasado tuvo enorme relevancia económica y social y que en la actualidad se considera un instrumento favorecedor del contacto del hombre con la naturaleza y de la ordenación del entorno medioambiental que precisa un gran esfuerzo para su conservación y defensa en la Región de Murcia.

Aun cuando la Ley 3/1995, de 23 de marzo, de Vías Pecuarias, en su condición de legislación básica, deja un espacio a la ordenación autonómica de desarrollo, acometida en numerosas comunidades autónomas, la Región de Murcia continúa en la actualidad sin hacer ejercicio de dicha competencia, pese a lo cual se han podido salvaguardar los valores propios de las vías pecuarias mediante los procedimientos de clasificación, deslinde y amojonamiento sin tener que demorar o supeditar su actuación a la aprobación de una norma autonómica en materia de vías pecuarias. 
Visto lo anterior, la presente Instrucción pretende facilitar un manual de aplicación de la normativa vigente ${ }^{19}$ que, teniendo en cuenta los criterios jurisprudenciales establecidos, pueda resultar de utilidad a los empleados públicos respecto de los procedimientos y las prácticas administrativos que se siguen para la clasificación, el deslinde y el amojonamiento de las vías pecuarias de la Región, permitiendo el ejercicio de las potestades de defensa y protección en este ámbito, incluyendo el procedimiento a seguir para el caso de la necesaria revisión o actualización de las clasificaciones preexistentes que así lo requieran para su adaptación a la realidad documental o a la de los terrenos. Todo lo anterior puede resultar de gran importancia a la vista del uso medioambiental, además del tradicional ganadero y agrícola, a que con carácter complementario viene destinado este dominio público.

\section{Turismo}

En relación con el ecoturismo y el turismo rural, debe tenerse en cuenta la nueva Ley 12/2013, de 20 de diciembre, de Turismo de la Región de Murcia. Como es sabido, la industria turística es sin duda un sector estratégico fundamental de la economía de la Región de Murcia. Si bien las históricas deficiencias en la red de infraestructuras de la Región de Murcia han impedido que el turismo alcance el nivel de desarrollo que merece por su especial ubicación en la costa mediterránea. El propósito de esta ley es facilitar una solución a tales deficiencias posibilitando que la Región sea un destino más accesible para el turismo. Si bien, y como la propia norma reconoce, la mejora de las infraestructuras, pese a ser importante, no es suficiente. Es necesario facilitar la inversión productiva. Por ello, deviene necesario modificar el marco legislativo actual recogido en la Ley 11/1997, de 12 de diciembre, de Turismo de la Región de Murcia, y establecer una nueva ley que elimine los obstáculos y facilite los trámites, adaptándose mejor a la innovación empresarial.

Desde el punto de vista ambiental podemos destacar la inclusión entre los principios rectores de la norma del valor del turismo como fuente de conocimiento cultural y de

\footnotetext{
${ }^{19}$ Constituida principalmente por la Ley 3/1995, de 23 de marzo, de Vías Pecuarias, la Ley estatal 33/2003, de 3 de noviembre, del Patrimonio de las Administraciones Públicas y su Reglamento, la Ley regional 3/1992, de 30 de julio, de Patrimonio de la Región de Murcia, y la Ley 30/1992, de 26 de noviembre, de Régimen Jurídico de las Administraciones Públicas y del Procedimiento Administrativo Común.
} 
sostenibilidad ambiental y territorial (art. 4.5) y el fomento del ecoturismo y el turismo rural, esto es, turismo relacionado con los recursos naturales de la Región, implementando acciones tendentes a ello con sujeción al ordenamiento jurídico medioambiental (art. 12).

\section{Evaluación ambiental de proyectos}

Aquí hay que mencionar el expediente relativo al Parque Temático Paramount. En relación con dicha cuestión, se ha hecho pública la Resolución de la Dirección General de Medio Ambiente por la que se adopta la decisión de no someter al procedimiento de evaluación ambiental de proyectos el Parque Temático Paramount, en el término municipal de Alhama de Murcia, a solicitud de Premursa Theme Park, S. A., con número de expediente 66/13 AU/AAU (CIF: A-73686263) ${ }^{20}$.

El pasado 14 de febrero se hizo pública en el BORM número 37 la decisión del director general de Medio Ambiente de no someter a evaluación ambiental el proyecto de Parque Temático Paramount, en el término municipal de Alhama de Murcia. Se adopta esta decisión pese a que parte de su superficie - 3,36 hectáreas ocupadas por la zona de aparcamientos - está incluida en el Parque Regional El Valle-Carrascoy ${ }^{21}$, si bien fuera del ámbito del PORN aprobado inicialmente por Orden de 18 de mayo de 2005 de la Consejería de Industria y Medio Ambiente.

Con carácter previo a la adopción de esta decisión han sido consultadas las administraciones, personas e instituciones $\operatorname{afectadas}^{22}$, de conformidad con lo establecido en el artículo 17 del Real Decreto Legislativo 1/2008, de 11 de enero, por el que se aprueba el texto refundido de la Ley de Evaluación de Impacto Ambiental de Proyectos, y en el artículo 85 de la Ley regional 4/2009, de 14 de mayo, de Protección Ambiental Integrada.

Los informes emitidos por la Dirección General de Carreteras -informe de 20 de septiembre-, el Instituto de Turismo -informe de 24 de septiembre- o el

\footnotetext{
${ }^{20}$ El contenido completo de la decisión está disponible en la página web $<$ http://www.carm.es/>.

${ }^{21}$ Según delimitación establecida en la Ley de 4/1992, de 30 de julio, de Ordenación y Protección del Territorio de la Región de Murcia, Sentencia del Tribunal Constitucional de 13 de diciembre de 2012.

${ }^{22}$ Entre ellas, el Ayuntamiento de Alhama de Murcia; la Confederación Hidrográfica del Segura; la Delegación del Gobierno; seis direcciones generales de diferentes consejerías; la Consejería de Cultura y Turismo; la Asociación de Naturalistas del Sureste; y Ecologistas en Acción.
} 
Ayuntamiento de Alhama —informe técnico de 10 de octubre-, entre otros, sostienen que el Parque Temático no tendrá efectos negativos en el medio ambiente. Sin embargo, las asociaciones conservacionistas consultadas, entre ellas Ecologistas en Acción informe de 22 de octubre de 2013-, mantienen el necesario sometimiento del Parque Temático a una evaluación de impacto ambiental de proyectos ya que que el documento ambiental presentado solo describe someramente y minimiza los aspectos ambientales que tienen efectos negativos relevantes que requieren el mecanismo del proceso previsto en el contexto del artículo 85 de la Ley 4/2009, de 14 de mayo, de Protección Ambiental Integrada de la Región de Murcia.

\section{Ordenanzas locales}

En relación con las ordenanzas locales en materia de medio ambiente, deben citarse las siguientes:

- Totana (aprobación inicial de la Ordenanza Municipal reguladora de la instalación y funcionamiento de infraestructuras radioeléctricas, BORM, núm. 4, de 7 de enero de 2014). La incidencia que muchas de estas instalaciones tienen en el paisaje urbano y rural, junto con el necesario acceso de los ciudadanos a estos servicios de telecomunicaciones, justifica la elaboración y aprobación por parte del Ayuntamiento de Totana de una ordenanza municipal propia reguladora del impacto urbanístico y medioambiental sobre el territorio municipal de dichas infraestructuras, que se someten al correspondiente régimen de licencias. Esta nueva ordenanza establece una serie de requisitos que deberán cumplir este tipo de instalaciones tanto respecto a la regulación de las condiciones urbanísticas, de protección ambiental y de seguridad, como respecto al sometimiento a licencia de su implantación y funcionamiento.

- Mazarrón (aprobación definitiva de la Ordenanza Municipal para la prevención y control de los mosquitos y particularmente del mosquito tigre, BORM, núm. 24, de 30 de enero de 2014). La presencia del mosquito tigre (Aedes albopictus) tiene serias implicaciones medioambientales y sanitarias por ser una especie invasora e importante vector de diversas enfermedades víricas. La experiencia demuestra que esta especie de mosquito se adapta muy bien a las peculiaridades ecológicas del área mediterránea, colonizando nuevos hábitats y criando en pequeñas masas de agua, naturales o artificiales, estancadas y cerradas como bidones, jarras, platos de macetas, cubos, 
neumáticos u otros tipos de recipientes que les recuerdan su hábitat natural, constituido por agujeros inundados en el tronco de los árboles.

Aunque este mosquito es vector de varias enfermedades en algunos países, ni en España ni en la Región de Murcia se le puede considerar un transmisor. Pero es una especie foránea de mosquito que hay que controlar para prevenir los efectos nocivos que pudiera llegar a causar en nuestra población. Es una especie diurna y muy activa que produce múltiples molestias y dolorosas picaduras.

Esta ordenanza municipal pretende aportar la información, las soluciones y los consejos necesarios a los ciudadanos para evitar la presencia y/o expansión de los mosquitos en el municipio y dotar al Ayuntamiento de las herramientas necesarias para hacer cumplir las medidas oportunas en aquellos espacios que puedan contener aguas estancadas, así como para permitir a los órganos competentes sancionar a los sujetos responsables de la reproducción del mosquito tigre o de las otras especies de mosquitos autóctonos.

- Molina de Segura (aprobación definitiva de la modificación de la Ordenanza reguladora de la emisión de ruidos y vibraciones, BORM, núm. 26, de 1 de febrero de 2014). Desde el punto de vista ambiental, la Orden tipifica como infracciones las acciones y omisiones que vulneren las prescripciones contenidas en la legislación medioambiental.

- Torre Pacheco (Resolución de la Alcaldía de Torre Pacheco, de fecha 4 de marzo de 2014, por la que se acuerda la aprobación definitiva de la Ordenanza de Limpieza Viaria y Gestión de Residuos). La presente Ordenanza regula las situaciones y actividades dirigidas al mantenimiento de la limpieza de los espacios públicos y privados, así como la gestión de los residuos domésticos, a fin de proteger el medio ambiente, el ornato público y la presencia urbana, de acuerdo con las competencias que corresponden al Ayuntamiento de Torre Pacheco. Esta ordenanza es de aplicación exclusivamente a los residuos domésticos, según la definición recogida en la Ley 22/2011, de 28 de julio, de Residuos y Suelos Contaminados. 